\title{
Noise Response and Stability in Injection Locked Semiconductor Ring Laser
}

\author{
M.I. MEMOn AND H. FAThallah
}

Prince Sultan Advanced Technologies Research Institute (PSATRI), King Saud University, Riyadh, Saudi Arabia

\begin{abstract}
Stability and effects of optical injection locking in semiconductor ring laser is modeled in detail. It is verified that the injection locking in slave semiconductor ring laser depends on detuning frequency and external optical injection ratio between the master laser and the slave semiconductor ring laser. The stability of injection was locked using the resonance frequency and damping factor. The parasitic phase modulation response due to amplitude modulation (chirp response) is derived and simulated. Similarly parasitic amplitude modulation due to phase modulation response is also investigated.
\end{abstract}

DOI: $10.12693 /$ APhysPolA.125.454

PACS: 42.55.Wd, 42.65.Re, 42.60.By, 42.65.Pc

\section{Introduction}

High speed laser sources can fulfill the transmission demands for next-generation communication networks. In the master-slave configuration, optical injection locking (OIL) has proven its potential to enhance the modulation bandwidth of a slave semiconductor laser to an extent much greater than its original bandwidth [1, 2]. Semiconductor ring laser (SRL) as the slave laser is a robust device for coplanar integration as it works in the direction of external injection. Consequently, optical isolator is not required between the master and slave lasers.

Previously, we have already demonstrated enhancement in the modulation bandwidth of SRL both experimentally and theoretically.

In this paper, stability of optical injection locking in SRL is discussed. It is also attempted to derive an expression for the frequency response (chirp response) of parasitic modulation in the output phase of OIL-SRL due to change in the intensity modulated input signal.

Frequency chirp is the instantaneous change in the frequency of modulated output light of the laser. It is one of the most severe limitations along with the fiber chromatic dispersion to the maximum attainable value of the length-bit rate product in the data transmissions at $1550 \mathrm{~nm}$ wavelength [3]. Although injection locking systems have provided sufficient reduction in the chirp [4-6] for high speed communication systems, most of the research is concentrated on the direct modulation in injection locked semiconductor lasers [5]. However, in the intensity modulation (amplitude modulation), the significant measure that affects the system performances is the ratio between the fundamental frequency and the intensity modulation photon densities [4].

\section{Theory}

SRL is a bistable laser device that when it is operated in the uni-direction region lases in the direction of external injection. When the SRL is locked by the light from the master laser in the $\mathrm{CCW}$ (counter clockwise) direction, it can be defined by following set single mode rate equations as in [2]. By linearizing these rate equations may be placed

$$
\left[\begin{array}{l}
\delta S \\
\delta \varphi \\
\delta N
\end{array}\right]=\boldsymbol{M}^{-1} \boldsymbol{X}
$$

where

$$
\boldsymbol{M}=\left[\begin{array}{ccc}
a_{11}+\mathrm{j} \omega & a_{12} & a_{13} \\
a_{21} & a_{22}+\mathrm{j} \omega & a_{23} \\
a_{31} & a_{32} & a_{33}+\mathrm{j} \omega
\end{array}\right] .
$$

The state transition matrix $M$ can be given as $a_{11}=$ $z \cos \varphi_{0}, a_{12}=2 z S_{0} \sin \varphi_{0}, a_{13}=-\Gamma v_{\mathrm{g}} g_{n} S_{0}, a_{21}=$ $-z \sin \varphi_{0} / 2 S_{0}, a_{22}=z \cos \varphi_{0}, a_{23}=-\alpha \Gamma v_{\mathrm{g}} g_{n} / 2, a_{31}=$ $\left(1 / \tau_{p}-2 z \cos \varphi_{0}\right) / \Gamma, a_{32}=0$ and $a_{33}=1 / \tau_{\mathrm{Nd}}+v_{\mathrm{g}} g_{n} S_{0}$, where $S_{0}, \varphi_{0}, N_{0}$, are steady-state values of photon density, output phase of SRL and carrier density, respectively. Similarly, the parameters $\alpha, \Gamma, v_{\mathrm{g}}, g_{n}, N, N_{\mathrm{tr}}$, $\varepsilon_{s}, \tau_{p}, \eta_{i}, I, q$, and $\tau_{\mathrm{Nd}}$ are line-width enhancement factor, optical confinement factor, group velocity, differential gain at transparency, carrier density, carrier density at transparency, self-gain saturation coefficient, differential photon life-time, the quantum efficiency of the bias current $I$, the electron charge in the volume of active region $V$ and carrier lifetime, respectively.

$\kappa_{\text {inj }}, S_{\text {injo }}$ account for the field coupling coefficient and initial photon density of injection laser, respectively.

It is attempted to derive expression for the frequency response of parasitic modulation in the output phase of OIL-SRL due to change in the amplitude modulated input signal. When amplitude modulated signal is injected into the SRL, the input vector $X$ can be described as

$$
\boldsymbol{X}_{\mathrm{AM}}=\left[\begin{array}{c}
z S_{0} / S_{\mathrm{inj} 0} \cos \varphi_{0} \\
-z \sin \varphi_{0} / 2 S_{\mathrm{inj} 0} \\
0
\end{array}\right] \delta S_{\mathrm{inj}}
$$

So the frequency response for chirp to input modulated power can be given as by using Eq. (2) in Eq. (1) and 
normalized by multiplying modulation frequency $\omega$ :

$$
\begin{aligned}
& H_{\text {chirp }}(\mathrm{j} \omega)=\omega \frac{\delta \varphi}{\delta S_{\text {inj }}} \\
& \quad=M_{\text {chirp }} \omega \frac{\omega^{2} a_{1}-\mathrm{j} \omega b_{1}+c_{1}}{-\mathrm{j} \omega^{3}-\omega^{2} A+\mathrm{j} \omega B+C},
\end{aligned}
$$

where

$$
\begin{aligned}
& M_{\text {chirp }}=\frac{z}{2 S_{\text {injo }}}, \\
& a_{1}=\sin \varphi_{0}, \\
& b_{1}=\left(a_{11}+a_{33}\right) \sin \varphi_{0}+2 a_{21} S_{0} \cos \varphi_{0}, \\
& c_{1}=\left(a_{31} a_{13}-a_{11} a_{33}\right) \sin \varphi_{0} \\
& \quad+2 S_{0}\left(a_{31} a_{23}-a_{21} a_{33}\right) \cos \varphi_{0} .
\end{aligned}
$$

\section{Simulation results and discussion}

Chirp response is simulated at different injection power and the detuning frequency is kept constant to $3.5 \mathrm{GHz}$ as shown in Fig. 1. As discussed earlier, the resonance frequency and the damping factor increase with the increase in the injection ratio. Chirp response becomes almost flat and low in amplitude at high injection ratio.

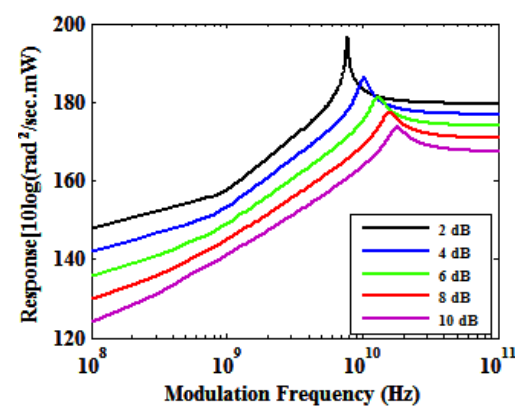

Fig. 1. Normalised chirp response with change in injection ratio.

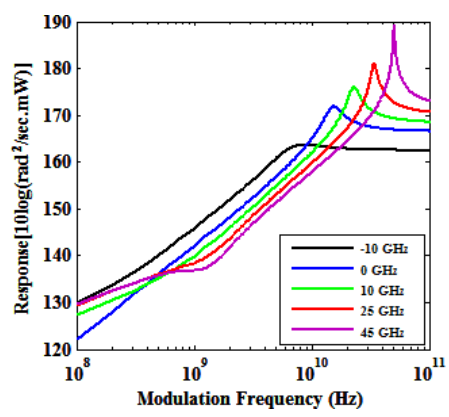

Fig. 2. Normalised chirp response with change in detuning frequency.
Figure 2 shows the chirp response at different detuning frequencies and fixed injection ratio of $10 \mathrm{~dB}$, the resonance becomes narrow and enhanced with increase in detuning frequency. It is due to the fact that the damping factor decreases with the increase in the detuning frequency from the negative to the positive edge of the locking range.

\section{Conclusion}

In this paper, we have derived and simulated the expressions for the chirp response that is defined as the parasitic phase modulation response due to amplitude modulation. They are found to be not very high at low modulation frequencies but with the increase in the resonance frequency, there is resonance in both the responses, respectively. This scheme may lead to a low-cost source in optical communication systems with high speed.

\section{References}

[1] M.I. Memon, B. Li, G. Mezosi, Z. Wang, M. Sorel, S. Yu, IEEE Photon. Techn. Lett. 21, 1792 (2009).

[2] M.I. Memon, H. Fathallah, S. Yu, Acta Phys. Pol. A 123, 180 (2013).

[3] J.M. Osterwalder, B.J. Rickett, IEEE J. Quant. Electron. 16, 250 (1980).

[4] T.L. Koch, J.E. Bowers, Electron. Lett. 20, 1038 (1984).

[5] S. Piazzolla, P. Spano, M. Tamburrini, IEEE J. Quant. Electron. 22, 2219 (1986).

[6] G. Yabre, IEEE J. Light Wave Technol. 14, 2367 (1996). 